\title{
ON AREA STATIONARY SURFACES IN THE SPACE OF ORIENTED GEODESICS OF HYPERBOLIC 3-SPACE
}

\author{
NIKOS GEORGIOU*
}

\begin{abstract}
We study area-stationary surfaces in the space $\mathrm{L}\left(\mathrm{H}^{3}\right)$ of oriented geodesics of hyperbolic 3-space, endowed with the canonical neutral Kähler structure. We prove that every holomorphic curve in $\mathrm{L}\left(\mathrm{H}^{3}\right)$ is an area-stationary surface. We then classify Lagrangian area-stationary surfaces $\Sigma$ in $\mathrm{L}\left(\mathrm{H}^{3}\right)$ and prove that the family of parallel surfaces in $\mathrm{H}^{3}$ orthogonal to the geodesics $\gamma \in \Sigma$ form a family of equidistant tubes around a geodesic. Finally we find an example of a two parameter family of rotationally symmetric area-stationary surfaces that are neither Lagrangian nor holomorphic.
\end{abstract}

The last two decades has seen increasing interest in spaces $L(M)$ of oriented geodesics of a manifold $\mathrm{M}$, with particular attention to their rich geometric structure. In the case of the space $L\left(E^{3}\right)$ of oriented affine lines in Euclidean 3 -space this interest can be traced back over a hundred years to Weierstrass's construction of minimal surfaces [16] and Whittaker's solutions to the wave equation [17].

A natural complex structure on $\mathrm{L}\left(\mathrm{E}^{3}\right)$ was considered by Hitchin to construct monopoles in $E^{3}$ [11], and then Guilfoyle and Klingenberg understood that the canonical symplectic structure on $L\left(E^{3}\right)$ is compatible with this complex structure [7], [8] and that the associated Kähler metric is of neutral signature. Salvai subsequently proved that this neutral Kähler metric is (up to addition of the round metric) the unique metric on $\mathrm{L}\left(\mathrm{E}^{3}\right)$ that is invariant under Euclidean motions [12]. This Kähler structure has recently been used by Guilfoyle and Klingenberg to solve an 80 year old conjecture of Carathéodory [10].

More recently, Anciaux, Guilfoyle and Romon [2] have studied Lagrangian area-stationary surfaces in $T N$, with $N$ being an oriented Riemannian surface and the neutral Kähler structure generalising that of the space of oriented geodesics in Euclidean and Lorentzian 3-space.

In addition, Salvai constructed a neutral Kähler metric on the space $\mathrm{L}\left(\mathrm{H}^{3}\right)$ of oriented geodesics in hyperbolic 3-space [13], while the geometry of $\mathrm{L}\left(\mathrm{H}^{3}\right)$

\footnotetext{
* The author is supported by FAPESP Foundation for Research Support of the State of São Paulo, Brazil.

Received 18th January 2011.
} 
was explored by Georgiou, Guilfoyle and Klingenberg [4], [5], [6].

Recently Alekseevsky, Guilfoyle and Klingenberg have given a description of all metrics defined on the space $L(M)$ of oriented geodesics of space forms or rank 1 Riemannian symmetric spaces, that are invariant under the isometry group of M [1].

The current paper can be considered as a continuation of the work of Georgiou and Guilfoyle (see [6]) on surface theory of $\mathrm{L}\left(\mathrm{H}^{3}\right)$. Here we study immersed area stationary surfaces, that is, immersed surfaces that area stationary for the area functional.

Since $\mathrm{L}\left(\mathrm{H}^{3}\right)=S^{2} \times S^{2}-\{$ diag $\}$, we can consider surfaces in $\mathrm{L}\left(\mathrm{H}^{3}\right)$ of rank 0,1 or 2 , depending on the rank of the projection onto the first factor.

In the first two sections we illustrate the geometric background on the construction of $\mathrm{L}\left(\mathrm{H}^{3}\right)$ and its submanifold theory, including geodesics and surfaces (further details can be found in [4], [5], [6]).

In section 3 we investigate rank 1 surfaces in $\mathrm{L}\left(\mathrm{H}^{3}\right)$ and prove that there are no holomorphic curves of rank 1. Moreover, in the case of a surface being Lagrangian of rank 1, we prove that it can not be area stationary.

Surfaces of rank 2 are studied in section 4 and, in particular, we prove that every holomorphic curve of rank 2 is area stationary and, since the only holomorphic curve of rank 0 are orthogonal to a horosphere, we conclude that every holomorphic curve is area stationary.

We also classify all Lagrangian area stationary surfaces. We summarize this result as:

MaIN Theorem. Let $S \subset \mathrm{H}^{3}$ be a $C^{3}$ smooth immersed oriented surface and $\Sigma \subset \mathrm{L}\left(\mathrm{H}^{3}\right)$ be the Lagrangian surface formed by the oriented geodesics normal to $S$.

The surface $\Sigma$ is area stationary iff $S$ is an equidistant tube around a geodesic in $\mathrm{H}^{3}$. In terms of holomorphic coordinates $\left(\mu_{1}, \mu_{2}\right)$ on $\mathrm{L}\left(\mathrm{H}^{3}\right)$, the surface $\Sigma$ is given by

$$
\bar{\mu}_{2}=\frac{1+\lambda_{1} \mu_{1}}{\lambda_{2}+\mu_{1}},
$$

where $\lambda_{1}, \lambda_{2} \in \mathrm{C}$ with $\lambda_{1} \lambda_{2} \neq 1$.

Finally, in section 5 we obtain a two parameter family of area stationary rotationally symmetric surfaces that are neither Lagrangian nor holomorphic.

\section{The Neutral Kähler Metric on $L\left(H^{3}\right)$}

We now describe the construction of the canonical Kähler metric on the space $\mathrm{L}\left(\mathrm{H}^{3}\right)$ of oriented geodesics in Hyperbolic 3-space - further details can be found in [4]. 
Consider the four dimensional manifold $\mathrm{S}^{2} \times \mathrm{S}^{2}$ endowed with the canonical complex structure $\mathrm{J}=j \oplus j$. Here, and throughout, we write $\mathrm{P}^{1}$ for $\mathrm{S}^{2}$ with the standard complex structure. By taking local holomorphic coordinates $\left(\mu_{1}, \mu_{2}\right)$, we set $\bar{\Delta}=\left\{\left(\mu_{1}, \mu_{2}\right) \in \mathrm{P}^{1} \times \mathrm{P}^{1} \mid \mu_{1} \bar{\mu}_{2}=-1\right\}$. Then $\mathrm{L}\left(\mathrm{H}^{3}\right)$ can be identified with $\mathrm{P}^{1} \times \mathrm{P}^{1}-\bar{\Delta}$.

The Kähler metric $\mathrm{G}$ and the symplectic form $\Omega$ on $\mathrm{L}\left(\mathrm{H}^{3}\right)$ are expressed locally by:

$$
\mathrm{G}=\operatorname{Im}\left(\frac{2}{\left(1+\mu_{1} \bar{\mu}_{2}\right)^{2}} d \mu_{1} \otimes d \bar{\mu}_{2}\right), \quad \Omega=-\operatorname{Re}\left(\frac{2}{\left(1+\mu_{1} \bar{\mu}_{2}\right)^{2}} d \mu_{1} \wedge d \bar{\mu}_{2}\right) .
$$

It has been proven that the Kähler metric $\mathrm{G}$ is scalar flat, conformally flat and is of signature $(++--)$.

Moreover, $\mathrm{G}$ is invariant under the action induced on $\mathrm{L}\left(\mathrm{H}^{3}\right)$ by the isometry group of $\mathrm{H}^{3}$. Indeed, this has been shown to be the unique Kähler metric on $\mathrm{L}\left(\mathrm{H}^{3}\right)$ with this property [13].

In order to transfer geometric data between $\mathrm{L}\left(\mathrm{H}^{3}\right)$ and $\mathrm{H}^{3}$ we use the correspondence space:

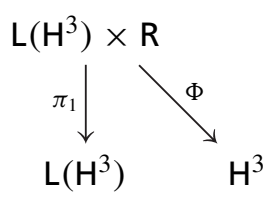

Given $\gamma \in \mathrm{L}\left(\mathrm{H}^{3}\right)$, the set $\Phi \circ \pi_{1}^{-1}(\gamma)$ is the oriented geodesic in $\mathrm{H}^{3}$, while, for a point $p \in \mathrm{H}^{3}, \pi_{1} \circ \Phi^{-1}(p)$ is the set of oriented geodesics in $\mathrm{L}\left(\mathrm{H}^{3}\right)$ that pass through $p$.

The map $\Phi$ takes an oriented geodesic $\gamma$ in $\mathrm{L}\left(\mathrm{H}^{3}\right)$ and a real number $r$ to the point on $\gamma$ an affine parameter distance $r$ from some fixed point on the geodesic. This choice of point on each geodesic can be made globally, but we more often just use a local choice, which is sufficient for our purposes.

Let $\left(x_{0}, x_{1}, x_{2}\right)$ be the local coordinates of the upper-half space model of $\mathrm{H}^{3}$. Then, in terms of holomorphic coordinates $\left(\mu_{1}, \mu_{2}\right)$ on $\mathrm{L}\left(\mathrm{H}^{3}\right)$ the map $\Phi$ has expression:

$$
z=\frac{1-\mu_{1} \bar{\mu}_{2}}{2 \bar{\mu}_{2}}+\left(\frac{1+\mu_{1} \bar{\mu}_{2}}{2 \bar{\mu}_{2}}\right) \tanh r, \quad t=\frac{\left|1+\bar{\mu}_{1} \mu_{2}\right|}{2\left|\mu_{2}\right| \cosh r},
$$

where $z=x_{1}+i x_{2}$ and $t=x_{0}$.

\section{Surfaces in $\mathrm{L}\left(\mathrm{H}^{3}\right)$}

Our interest in this paper is focused on the study of two parameter families of oriented geodesics, or surfaces in $\mathrm{L}\left(\mathrm{H}^{3}\right)$. Therefore, we recall some basic 
results on the surface theory of the space of oriented geodesics in hyperbolic 3 -space - further details can be found in [6].

In order to avoid any confusion, whether we use the expression of geodesic congruence we mean a surface in $\mathrm{L}\left(\mathrm{H}^{3}\right)$.

For computational purposes, we give explicit local parameterizations of the geodesic congruence. The dual picture of a surface in $\mathrm{L}\left(\mathrm{H}^{3}\right)$ is to consider the surfaces in $\mathrm{H}^{3}$ that are orthogonal to a given set of geodesics. However, not every geodesic congruence has such orthogonal surfaces - indeed, most don't. To explain this further, we consider the first order properties of $\Sigma$, which can be described by two complex functions, the optical scalars: $\rho, \sigma: \Sigma \times \mathrm{R} \mapsto \mathrm{C}$. The real part $\Theta$ and imaginary part $\lambda$ of $\rho$ are the divergence and twist of the geodesic congruence, while $\sigma$ is the shear.

Given a surface $\Sigma \subset \mathrm{L}\left(\mathrm{H}^{3}\right)$, a trio $\left\{e_{0}, e_{+}, e_{-}\right\}$of complex vector fields in $\mathrm{C} \otimes T \mathrm{H}^{3}$ is said to be an adapted null frame if for each $\gamma \in \Sigma$, we have $e_{0}=\dot{\gamma}$ and $e_{+}$is the complex conjugate of $e_{-}$such that the orientation of $\left\{e_{0}, \operatorname{Re}\left(e_{+}\right), \operatorname{Im}\left(e_{+}\right)\right\}$is the standard orientation on $\mathrm{H}^{3}$, and:

$$
\left\langle e_{0}, e_{0}\right\rangle=\left\langle e_{+}, e_{-}\right\rangle=1, \quad\left\langle e_{0}, e_{+}\right\rangle=\left\langle e_{+}, e_{+}\right\rangle=0 .
$$

The optical scalars are defined by:

$$
\rho=\left\langle\nabla_{e_{-}} e_{+}, e_{0}\right\rangle, \quad \sigma=\left\langle\nabla_{e_{+}} e_{+}, e_{0}\right\rangle,
$$

where $\nabla$ denotes the Levi-Civita connection of the hyperbolic metric.

In terms of the holomorphic coordinates $\left(\mu_{1}, \mu_{2}\right)$, the optical scalars have the following local expressions:

$$
\begin{aligned}
\sigma & =\frac{8 \mu_{2} J_{\overline{2} \overline{1}}}{\bar{\mu}_{2} \Delta\left|1+\mu_{1} \bar{\mu}_{2}\right|^{2}} \\
\rho & =-1-\frac{8 e^{-r}}{\Delta}\left[\frac{J_{2 \overline{1}}}{\left(1+\bar{\mu}_{1} \mu_{2}\right)^{2}} e^{r}-\frac{\left|\mu_{2}\right|^{2} J_{1 \overline{1}}}{\left|1+\bar{\mu}_{1} \mu_{2}\right|^{2}} e^{-r}\right],
\end{aligned}
$$

where

$$
J_{k l}=\partial \mu_{k} \bar{\partial} \mu_{l}-\bar{\partial} \mu_{k} \partial \mu_{l}
$$

and

$$
\begin{aligned}
\frac{1}{4} \Delta=\frac{J_{2 \overline{2}}}{\left|\mu_{2}\right|^{2}\left|1+\mu_{1} \bar{\mu}_{2}\right|^{2}} e^{2 r}+ & \left.\frac{J_{\overline{2} 1}}{(1}+\mu_{1} \bar{\mu}_{2}\right)^{2} \\
& \quad+\frac{J_{\overline{1} 2}}{\left(1+\bar{\mu}_{1} \mu_{2}\right)^{2}}+\frac{\left|\mu_{2}\right|^{2} J_{1 \overline{1}}}{\left|1+\mu_{1} \bar{\mu}_{2}\right|^{2}} e^{-2 r} .
\end{aligned}
$$


A surface $\Sigma$ in $\mathrm{L}\left(\mathrm{H}^{3}\right)$, is said to be Lagrangian if the symplectic form $\Omega$ pulled back to $\Sigma$ vanishes. We now give the following Proposition:

Proposition 1 ([6]). The following statements are equivalent:

(i) the geodesic congruence $\Sigma$ is Lagrangian,

(ii) locally there exists an embedded surface $S$ in $\mathrm{H}^{3}$ such that the geodesics of $\Sigma$ are normal to $S$,

(iii) the imaginary part $\lambda$ of $\rho$ (the twist) is zero.

Let $\Sigma$ be a Lagrangian surface in $\mathrm{L}\left(\mathrm{H}^{3}\right)$ parameterized by $v \mapsto\left(\mu_{1}(v, \bar{v})\right.$, $\left.\mu_{2}(\nu, \bar{v})\right)$. The surfaces $S$ in $\mathrm{H}^{3}$ orthogonal to the geodesics of $\Sigma$ are given by (1.1), where the functions $r=r(v, \bar{v})$ solve:

$$
2 \partial r=\frac{\mu_{2}}{\bar{\mu}_{1} \mu_{2}+1}\left(\partial \bar{\mu}_{1}+\frac{\partial \mu_{2}}{\mu_{2}^{2}}\right)+\frac{\bar{\mu}_{2}}{\mu_{1} \bar{\mu}_{2}+1}\left(\partial \mu_{1}+\frac{\partial \bar{\mu}_{2}}{\bar{\mu}_{2}^{2}}\right),
$$

where $\partial$ denotes the derivative with respect of $v$.

The set of Lagrangian geodesic congruences is divided into three categories, depending on the rank of the immersion of the geodesic congruence.

Definition 1. Given an immersion $f: \Sigma \rightarrow \mathrm{L}\left(\mathrm{H}^{3}\right)$, consider the map $(\pi \circ f)_{*}: T \Sigma \rightarrow T \mathrm{P}^{1}$, where $\pi$ is projection onto the first factor of $\mathrm{L}\left(\mathrm{H}^{3}\right)=$ $\mathrm{P}^{1} \times \mathrm{P}^{1}-\bar{\Delta}$. The rank of the immersion $f$ at a point $\gamma \in \Sigma$ is defined to be the rank of this map at $\gamma$, which can be 0,1 or 2 .

Note that by reversing the orientation of the geodesics, the rank can be defined by projection onto the second factor. A rank 0 Lagrangian geodesic congruence correspond to a geodesic congruence orthogonal to a horosphere.

In the Lagrangian case, the functions $\sigma$ and $\rho$ have the following interpretation in terms of the second fundamental form of the orthogonal surfaces in $\mathrm{H}^{3}$.

Proposition 2 ([6]). Let $S \subset \mathrm{H}^{3}$ be a $C^{2}$ immersed surface and $\Sigma \subset \mathrm{L}\left(\mathrm{H}^{3}\right)$ be the oriented normal geodesic congruence. Then

$$
|\sigma|=\frac{1}{2}\left|\lambda_{1}-\lambda_{2}\right|, \quad \rho=-\frac{1}{2}\left(\lambda_{1}+\lambda_{2}\right),
$$

where $\lambda_{1}$ and $\lambda_{2}$ are the principal curvatures of $S$.

The induced metric $\mathrm{G}_{\Sigma}$ on a Lagrangian surface $\Sigma$ in $\mathrm{L}\left(\mathrm{H}^{3}\right)$ can be described by the functions $\sigma$ and $\lambda$ :

THEOREM 1 ([6]). Let $\Sigma$ be a surface in $\mathrm{L}\left(\mathrm{H}^{3}\right)$. The induced metric is Lorentz (degenerate, Riemannian) iff $|\sigma|^{2}-\lambda^{2}>0(=0,<0)$, where $\lambda$ and $\sigma$ are the twist and the shear of $\Sigma$. 
The theorem says that if a surface is Lagrangian then is either Lorentz or degenerate, the latter occurring at umbilic points on the orthogonal surfaces in $\mathrm{H}^{3}$.

The following theorem recovers the Weierstrass representation for the flat surfaces in $\mathrm{H}^{3}$ :

THEOREM 2 ([6]). Let $S$ be an oriented $C^{2}$ smooth immersed surface in $\mathrm{H}^{3}$ with normal geodesic congruence $\Sigma$. Assume that the metric $\mathrm{G}_{\Sigma}$ induced on $\Sigma$ by the neutral Kähler metric is non-degenerate.

Then $S$ is flat iff $\Sigma$ is of rank two and is parameterized by $\mu_{2}=\mu_{2}\left(\bar{\mu}_{1}\right)$, that is, $\mu_{2}$ is an anti-holomorphic function of $\mu_{1}$.

We use the complex structure $\mathrm{J}$ of $\mathrm{L}\left(\mathrm{H}^{3}\right)$ in order to describe another important class of surfaces:

Definition 2. A point $\gamma$ on a surface $\Sigma \subset \mathrm{L}\left(\mathrm{H}^{3}\right)$ is said to be a complex point if the complex structure $\mathrm{J}$ acting on $\mathrm{L}\left(\mathrm{H}^{3}\right)$ preserves $T_{\gamma} \Sigma$. A surface $\Sigma \subset \mathrm{L}\left(\mathrm{H}^{3}\right)$ is said to be a holomorphic curve if all of the points of $\Sigma$ are complex points.

In particular:

PROPOSITION 3 ([6]). A point $\gamma$ on a surface $\Sigma$ is complex iff the shear vanishes along $\gamma$.

By Proposition 2, observe that complex points on a Lagrangian surface $\Sigma \subset \mathrm{L}\left(\mathrm{H}^{3}\right)$ correspond to umbilic points on the surfaces in $\mathrm{H}^{3}$ orthogonal to $\Sigma$.

\section{Non-existence of rank one area stationary surfaces}

Consider a surface $\Sigma \subset \mathrm{L}\left(\mathrm{H}^{3}\right)$ of rank one. Then $\Sigma$ can be locally parameterized by $\mu_{1}=\mu_{1}(s)$ and $\mu_{2}=\mu_{2}(s, t)$ where $(s, t) \in D$ with $D$ being an open subset of $\mathrm{R}^{2}$.

We have the following:

PROPOSITION 4. An immersed holomorphic curve in $\mathrm{L}\left(\mathrm{H}^{3}\right)$ cannot be of rank 1 .

Proof. Assume the existence of an immersed rank one surface on $\mathrm{L}\left(\mathrm{H}^{3}\right)$ such that in an open neighborhood $U \subset \Sigma$ is holomorphic, which, by Proposition 3, is equivalent to the vanishing of the shear $\sigma$ in this open set. Considering now the local parametrisation $\Sigma \rightarrow \mathrm{L}\left(\mathrm{H}^{3}\right):(s, t) \mapsto\left(\mu_{1}(s), \mu_{2}(s, t)\right)$, the vanishing of the shear implies the vanishing of $J_{\overline{2}} \overline{1}_{1}$, which means $\partial_{s} \bar{\mu}_{1} \partial_{t} \bar{\mu}_{2}=0$ on $U$. 
Then, in an open subset $V$ of $U$, either $\partial_{s} \bar{\mu}_{1}=0$ or $\partial_{t} \bar{\mu}_{2}=0$. In any case, we have a contradiction since, if $\partial_{s} \bar{\mu}_{1}=0$ then $\mu_{1}$ is constant and therefore $\Sigma$ is of rank 0 on $V$, and if $\partial_{t} \bar{\mu}_{2}=0$ then $\mu_{2}$ only depends on $s$ and $\Sigma$ would not be immersed.

We now assume that the rank 1 surface $\Sigma$ is Lagrangian. In this case the induced metric $g=f^{*} \mathrm{G}$ has components in local coordinates $(s, t)$ :

$$
g_{s s}=2 \operatorname{Im}\left[\frac{\partial_{s} \mu_{1} \partial_{s} \bar{\mu}_{2}}{\left(1+\mu_{1} \bar{\mu}_{2}\right)^{2}}\right], \quad g_{s t}=\operatorname{Im}\left[\frac{\partial_{s} \mu_{1} \partial_{t} \bar{\mu}_{2}}{\left(1+\mu_{1} \bar{\mu}_{2}\right)^{2}}\right], \quad g_{t t}=0,
$$

and the nonzero Christoffel symbols are given by:

$$
\begin{gathered}
\Gamma_{s s}^{s}=\operatorname{Re}\left(\frac{\partial_{s}^{2} \mu_{1}}{\partial_{s} \mu_{1}}-\frac{2 \bar{\mu}_{2} \partial_{s} \mu_{1}}{1+\mu_{1} \bar{\mu}_{2}}\right), \\
\Gamma_{s t}^{t}=\operatorname{Re}\left(\frac{\partial_{s t}^{2} \mu_{2}}{\partial_{t} \mu_{2}}-\frac{2 \bar{\mu}_{1} \partial_{s} \mu_{2}}{1+\bar{\mu}_{1} \mu_{2}}\right), \\
\Gamma_{t t}^{t}=\operatorname{Re}\left(\frac{\partial_{t}^{2} \mu_{2}}{\partial_{t} \mu_{2}}-\frac{2 \bar{\mu}_{1} \partial_{t} \mu_{2}}{1+\bar{\mu}_{1} \mu_{2}}\right) .
\end{gathered}
$$

It is already known that the induced metric $g$ of a rank one Lagrangian surface $\Sigma$ is scalar flat [6].

The second fundamental form $h=h_{i j}^{\mu_{k}}$ has non-vanishing components:

$$
\begin{aligned}
& h_{s s}^{\mu_{1}}=\partial_{s}^{2} \mu_{1}-\frac{2 \bar{\mu}_{2}\left(\partial_{s} \mu_{1}\right)^{2}}{1+\mu_{1} \bar{\mu}_{2}}-\partial_{s} \mu_{1} \Gamma_{s s}^{s}, \\
& h_{s s}^{\mu_{2}}=\partial_{s}^{2} \mu_{2}-\frac{2 \bar{\mu}_{1}\left(\partial_{s} \mu_{2}\right)^{2}}{1+\bar{\mu}_{1} \mu_{2}}-\partial_{s} \mu_{2} \Gamma_{s s}^{s}-\partial_{t} \mu_{2} \Gamma_{s s}^{t}, \\
& h_{s t}^{\mu_{2}}=\partial_{s t}^{2} \mu_{2}-\frac{2 \bar{\mu}_{1} \partial_{s} \mu_{2} \partial_{t} \mu_{2}}{1+\bar{\mu}_{1} \mu_{2}}-\partial_{t} \mu_{2} \Gamma_{s t}^{t}, \\
& h_{t t}^{\mu_{2}}=\partial_{t}^{2} \mu_{2}-\frac{2 \bar{\mu}_{1}\left(\partial_{t} \mu_{2}\right)^{2}}{1+\bar{\mu}_{1} \mu_{2}}-\partial_{t} \mu_{2} \Gamma_{t t}^{t},
\end{aligned}
$$

with $h_{i j}^{\bar{\mu}_{k}}=\overline{h_{i j}^{\mu_{k}}}$.

In contrast to what occurs in the space $\mathrm{L}\left(\mathrm{E}^{3}\right)$ of oriented lines in Euclidean 3-space [9], we have the following:

Proposition 5. There are no area stationary Lagrangian surfaces in $\mathrm{L}\left(\mathrm{H}^{3}\right)$ of rank one. 
Proof. Let $\Sigma$ be a Lagrangian surface in $\mathrm{L}\left(\mathrm{H}^{3}\right)$ of rank one, locally parameterized by $\mu_{1}=\mu_{1}(s)$ and $\mu_{2}=\mu_{2}(s, t)$.

The mean curvature vector $H=2 \operatorname{Re}\left(H^{\mu_{1}} \partial / \partial \mu_{1}+H^{\mu_{2}} \partial / \partial \mu_{2}\right)$ in local coordinates $(s, t)$ is given by:

$$
H^{\mu_{i}}=g^{s s} h_{s s}^{\mu_{i}}+2 g^{s t} h_{s t}^{\mu_{i}}+g^{t t} h_{t t}^{\mu_{i}}
$$

Then $H^{\mu_{1}}=0$ and it remains to find $H^{\mu_{2}}$. By using the expressions of $h_{i j}^{\mu_{i}}$ and by considering the Lagrangian condition:

$$
\frac{\partial_{s} \mu_{1} \partial_{t} \bar{\mu}_{2}}{\left(1+\mu_{1} \bar{\mu}_{2}\right)^{2}}=-\frac{\partial_{s} \bar{\mu}_{1} \partial_{t} \mu_{2}}{\left(1+\bar{\mu}_{1} \mu_{2}\right)^{2}}
$$

we find $H^{\mu_{2}}$, and finally the mean curvature vector $H$ of $\Sigma$ is:

$$
H=4 \operatorname{Re}\left[g^{s t} \frac{\left(1+\bar{\mu}_{1} \mu_{2}\right)^{2}}{\partial_{s} \bar{\mu}_{1}} \partial_{t}\left(\frac{\partial_{s} \mu_{1} \partial_{s} \bar{\mu}_{2}}{\left(1+\mu_{1} \bar{\mu}_{2}\right)^{2}}+\frac{\partial_{s} \bar{\mu}_{1} \partial_{s} \mu_{2}}{\left(1+\bar{\mu}_{1} \mu_{2}\right)^{2}}\right) \frac{\partial}{\partial \mu_{2}}\right],
$$

which means that the surface $\Sigma$ is area stationary iff

$$
\partial_{t}\left[\operatorname{Re}\left(\frac{\partial_{s} \mu_{1} \partial_{s} \bar{\mu}_{2}}{\left(1+\mu_{1} \bar{\mu}_{2}\right)^{2}}\right)\right]=0 .
$$

The above condition and the Lagrangian condition give:

$$
\begin{aligned}
A \partial_{s} \bar{\mu}_{2}+\bar{A} \partial_{s} \mu_{2} & =f(s), \\
A \partial_{t} \bar{\mu}_{2}+\bar{A} \partial_{t} \mu_{2} & =0,
\end{aligned}
$$

where

$$
A=\frac{\partial_{s} \mu_{1}}{\left(1+\mu_{1} \bar{\mu}_{2}\right)^{2}} .
$$

Differentiate equations (3.2) and (3.6) with respect to $t$ and $s$, respectively, and then subtract:

$$
\operatorname{Re}\left(\partial_{t} A \partial_{s} \bar{\mu}_{2}-\partial_{s} \bar{A} \partial_{t} \bar{\mu}_{2}\right)=0 .
$$

After a brief computation we get:

$$
\begin{aligned}
\partial_{s} A \partial_{t} \bar{\mu}_{2} & =\frac{\partial_{s}^{2} \mu_{1} \partial_{t} \bar{\mu}_{2}}{\left(1+\mu_{1} \bar{\mu}_{2}\right)^{2}}-\frac{2 \mu_{1} \partial_{s} \mu_{1} \partial_{s} \bar{\mu}_{2} \partial_{t} \bar{\mu}_{2}}{\left(1+\mu_{1} \bar{\mu}_{2}\right)^{3}}-\frac{2 \bar{\mu}_{2}\left(\partial_{s} \mu_{1}\right)^{2} \partial_{t} \bar{\mu}_{2}}{\left(1+\mu_{1} \bar{\mu}_{2}\right)^{3}}, \\
\partial_{t} A \partial_{s} \bar{\mu}_{2} & =-\frac{2 \mu_{1} \partial_{s} \mu_{1} \partial_{s} \bar{\mu}_{2} \partial_{t} \bar{\mu}_{2}}{\left(1+\mu_{1} \bar{\mu}_{2}\right)^{3}}
\end{aligned}
$$


and then condition (3.4) becomes

$$
\operatorname{Re}\left(\frac{2 \bar{\mu}_{2}\left(\partial_{s} \mu_{1}\right)^{2} \partial_{t} \bar{\mu}_{2}}{\left(1+\mu_{1} \bar{\mu}_{2}\right)^{3}}-\frac{\partial_{s}^{2} \mu_{1} \partial_{t} \bar{\mu}_{2}}{\left(1+\mu_{1} \bar{\mu}_{2}\right)^{2}}\right)=0 .
$$

Using the Lagrangian condition (3.1) in (3.5), we have

$$
\frac{\bar{\mu}_{2} \partial_{s} \mu_{1}}{1+\mu_{1} \bar{\mu}_{2}}-\frac{\mu_{2} \partial_{s} \bar{\mu}_{1}}{1+\bar{\mu}_{1} \mu_{2}}=\frac{1}{2}\left(\frac{\partial_{s}^{2} \mu_{1}}{\partial_{s} \mu_{1}}-\frac{\partial_{s}^{2} \bar{\mu}_{1}}{\partial_{s} \bar{\mu}_{1}}\right)=h(s) .
$$

Integration of (3.1) with respect of $t$ gives

$$
\frac{\bar{\mu}_{2} \partial_{s} \mu_{1}}{1+\mu_{1} \bar{\mu}_{2}}+\frac{\mu_{2} \partial_{s} \bar{\mu}_{1}}{1+\bar{\mu}_{1} \mu_{2}}=g(s),
$$

and then the sum $(3.6)+(3.7)$, is

$$
\frac{\bar{\mu}_{2} \partial_{s} \mu_{1}}{1+\mu_{1} \bar{\mu}_{2}}=h(s)+g(s)=m(s) \partial_{s} \mu_{1} .
$$

Hence

$$
\bar{\mu}_{2}=\frac{m}{1-m \mu_{1}}=\bar{\mu}_{2}(s),
$$

which is a contradiction, since $\Sigma$ is of rank one.

\section{Rank two area stationary surfaces}

Consider a rank 2 surface $\Sigma$ in $\mathrm{L}\left(\mathrm{H}^{3}\right)$. That is, a surface $\Sigma$ given locally by $\mu_{1} \rightarrow\left(\mu_{1}, \mu_{2}\left(\mu_{1}, \bar{\mu}_{1}\right)\right)$ for some smooth function $\mu_{2}: \mathrm{C} \rightarrow \mathrm{C}$. We are interested in area stationary surfaces in $\mathrm{L}\left(\mathrm{H}^{3}\right)$ of rank 2 and therefore we consider variations of the area integral

$$
\mathscr{A}(\Sigma)=\int_{\Sigma}|\mathrm{G}|^{\frac{1}{2}} d \mu_{1} d \bar{\mu}_{1} .
$$

For an arbitrary parameterization $\mu_{1} \rightarrow\left(\mu_{1}, \mu_{2}\left(\mu_{1}, \bar{\mu}_{1}\right)\right)$ the area integral is

$$
|G|=\frac{\Delta^{2}}{64}\left(\lambda^{2}-|\sigma|^{2}\right) \text {, }
$$

where

$$
\begin{aligned}
\lambda & =\frac{4 i}{\Delta}\left[\frac{\partial \mu_{2}}{\left(1+\bar{\mu}_{1} \mu_{2}\right)^{2}}-\frac{\bar{\partial} \bar{\mu}_{2}}{\left(1+\mu_{1} \bar{\mu}_{2}\right)^{2}}\right] \\
\sigma & =\frac{8 \mu_{2} \partial \bar{\mu}_{2}}{\bar{\mu}_{2} \Delta\left|1+\mu_{1} \bar{\mu}_{2}\right|^{2}}
\end{aligned}
$$


and

$$
\begin{aligned}
& \frac{1}{4} \Delta=\frac{\partial \mu_{2} \bar{\partial} \bar{\mu}_{2}-\bar{\partial} \mu_{2} \partial \bar{\mu}_{2}}{\left|\mu_{2}\right|^{2}\left|1+\mu_{1} \bar{\mu}_{2}\right|^{2}} e^{2 r} \\
& \quad-2 \operatorname{Re}\left(\frac{\partial \mu_{2}}{\left(1+\bar{\mu}_{1} \mu_{2}\right)^{2}}\right)+\frac{\left|\mu_{2}\right|^{2}}{\left|1+\mu_{1} \bar{\mu}_{2}\right|^{2}} e^{-2 r}
\end{aligned}
$$

with $\partial$ denotes the differentiation with respect to $\mu_{1}$.

A surface is area stationary if $\delta \mathscr{A}(\Sigma)=0$. In order to compute this quantity note that

$$
\frac{\Delta^{2} \lambda^{2}}{64}=-\frac{1}{4}\left[\frac{\partial \mu_{2}}{\left(1+\bar{\mu}_{1} \mu_{2}\right)^{2}}-\frac{\bar{\partial} \bar{\mu}_{2}}{\left(1+\mu_{1} \bar{\mu}_{2}\right)^{2}}\right]^{2}
$$

and so

$$
\begin{aligned}
& \delta\left(\frac{\Delta^{2} \lambda^{2}}{64}\right) \\
& \quad=-\operatorname{Re}\left[\frac{\partial \mu_{2}}{\left(1+\bar{\mu}_{1} \mu_{2}\right)^{2}}-\frac{\bar{\partial} \bar{\mu}_{2}}{\left(1+\mu_{1} \bar{\mu}_{2}\right)^{2}}\right]\left[\frac{\partial \delta \mu_{2}}{\left(1+\bar{\mu}_{1} \mu_{2}\right)^{2}}-\frac{2 \bar{\mu}_{1} \partial \mu_{2} \delta \mu_{2}}{\left(1+\bar{\mu}_{1} \mu_{2}\right)^{3}}\right],
\end{aligned}
$$

while, since

$$
\frac{\Delta^{2}|\sigma|^{2}}{64}=\frac{\partial \bar{\mu}_{2} \bar{\partial} \mu_{2}}{\left|1+\bar{\mu}_{1} \mu_{2}\right|^{4}}
$$

we have

$$
\delta\left(\frac{\Delta^{2}|\sigma|^{2}}{64}\right)=2 \operatorname{Re}\left(\frac{\partial \bar{\mu}_{2} \bar{\partial} \delta \mu_{2}}{\left|1+\bar{\mu}_{1} \mu_{2}\right|^{4}}-\frac{2 \bar{\mu}_{1}}{1+\bar{\mu}_{1} \mu_{2}} \frac{\partial \bar{\mu}_{2} \bar{\partial} \mu_{2} \delta \mu_{2}}{\left|1+\bar{\mu}_{1} \mu_{2}\right|^{4}}\right) .
$$

Combining these we find that

$$
\begin{aligned}
\delta|G|^{\frac{1}{2}}= & \frac{16}{\Delta \sqrt{\lambda^{2}-|\sigma|^{2}}} \operatorname{Re}\left\{-\frac{1}{2}\left[\frac{\partial \mu_{2}}{\left(1+\bar{\mu}_{1} \mu_{2}\right)^{2}}-\frac{\bar{\partial} \bar{\mu}_{2}}{\left(1+\mu_{1} \bar{\mu}_{2}\right)^{2}}\right]\right. \\
& \left.\cdot \partial\left(\frac{\delta \mu_{2}}{\left(1+\bar{\mu}_{1} \mu_{2}\right)^{2}}\right)-\frac{\partial \bar{\mu}_{2} \bar{\partial} \delta \mu_{2}}{\left|1+\bar{\mu}_{1} \mu_{2}\right|^{4}}+\frac{2 \bar{\mu}_{1}}{1+\bar{\mu}_{1} \mu_{2}} \frac{\partial \bar{\mu}_{2} \bar{\partial} \mu_{2} \delta \mu_{2}}{\left|1+\bar{\mu}_{1} \mu_{2}\right|^{4}}\right\} \\
= & 2 \operatorname{Re}\left[\frac{\lambda i}{\sqrt{\lambda^{2}-|\sigma|^{2}}} \partial\left(\frac{\delta \mu_{2}}{\left(1+\bar{\mu}_{1} \mu_{2}\right)^{2}}\right)-\frac{\bar{\mu}_{2} \sigma \bar{\partial} \delta \mu_{2}}{\mu_{2}\left|1+\bar{\mu}_{1} \mu_{2}\right|^{2} \sqrt{\lambda^{2}-|\sigma|^{2}}}\right. \\
& \left.+\frac{16 \bar{\mu}_{1}}{1+\bar{\mu}_{1} \mu_{2}} \frac{\partial \bar{\mu}_{2} \bar{\partial} \mu_{2} \delta \mu_{2}}{\Delta\left|1+\bar{\mu}_{1} \mu_{2}\right|^{4} \sqrt{\lambda^{2}-|\sigma|^{2}}}\right] .
\end{aligned}
$$

Integrating by parts we have established the following: 
PROPOSITION 6. A rank two surface is area stationary if and only if

$$
\begin{aligned}
& \frac{-i}{\left(1+\bar{\mu}_{1} \mu_{2}\right)^{2}} \partial\left(\frac{\lambda}{\sqrt{\lambda^{2}-|\sigma|^{2}}}\right) \\
& +\bar{\partial}\left(\frac{\bar{\mu}_{2} \sigma}{\mu_{2}\left|1+\bar{\mu}_{1} \mu_{2}\right|^{2} \sqrt{\lambda^{2}-|\sigma|^{2}}}\right)+\frac{\bar{\mu}_{1} \Delta|\sigma|^{2}}{4\left(1+\bar{\mu}_{1} \mu_{2}\right) \sqrt{\lambda^{2}-|\sigma|^{2}}}=0 .
\end{aligned}
$$

The following proposition shows that all holomorphic curves on $\mathrm{L}\left(\mathrm{H}^{3}\right)$ are area stationary:

Proposition 7. Every holomorphic curve $\Sigma$ in $\mathrm{L}\left(\mathrm{H}^{3}\right)$ such that the metric $\mathrm{G}_{\Sigma}$ induced on $\Sigma$ by the neutral Kähler metric is non-degenerate, is area stationary.

Proof. Consider a holomorphic curve $\Sigma$ in $\mathrm{L}\left(\mathrm{H}^{3}\right)$. Then by Proposition 3 the shear $\sigma$ vanishes throughout the surface $\Sigma$.

By Proposition 4 we know that a holomorphic surface $\Sigma$ can be either rank 0 or 2 . In the case of rank 0 , the surface $\Sigma$ is totally null and, in particular, it is orthogonal to a horosphere, which is not our case. Then $\Sigma$ must be of rank 2 and therefore Proposition 6 shows that is area stationary.

Consider now a Lagrangian surface $\Sigma$ in $\mathrm{L}\left(\mathrm{H}^{3}\right)$. We are interested in area stationary Lagrangian surfaces of rank 2 . In this case, the twist $\lambda$ vanishes on $\Sigma$ and then Proposition 6 implies that a Lagrangian surface $\Sigma$ of rank 2 will be area stationary iff

$$
\partial \ln \left(\frac{\bar{\sigma}_{0}}{\sigma_{0}}\right)-\frac{4 \bar{\mu}_{2}}{1+\mu_{1} \bar{\mu}_{2}}=0
$$

where

$$
\sigma_{0}=\frac{\partial \bar{\mu}_{2}}{\left(1+\mu_{1} \bar{\mu}_{2}\right)^{2}} .
$$

Definition 3. The Lagrangian angle $\phi$ of the surface $\Sigma \subset \mathrm{L}\left(\mathrm{H}^{3}\right)$ is defined by

$$
\sigma_{0}=\left|\sigma_{0}\right| e^{2 i \phi},
$$

where $\sigma_{0}$ is given by (4.5).

An equivalent condition that characterizes Lagrangian area stationary surfaces in $\mathrm{L}\left(\mathrm{H}^{3}\right)$ is given by the following proposition: 
Proposition 8. Let $\Sigma \subset \mathrm{L}\left(\mathrm{H}^{3}\right)$ be a Lagrangian surface of rank two. Then $\Sigma$ is a area stationary surface iff $\Sigma$ is locally orthogonal to a flat surface in $\mathrm{H}^{3}$ and the Lagrangian angle $\phi$, satisfies the following PDE:

$$
e^{-i \phi} \partial^{2} e^{-i \phi}=e^{i \phi} \bar{\partial}^{2} e^{i \phi}=\left|\sigma_{0}\right| \text {. }
$$

Proof. Assume that $\Sigma$ is a Lagrangian area stationary surface of rank two. Then $H^{\mu_{1}}=0$ which means that

$$
\partial \ln \left(\frac{\bar{\sigma}_{0}}{\sigma_{0}}\right)-\frac{4 \bar{\mu}_{2}}{1+\mu_{1} \bar{\mu}_{2}}=0
$$

and by introducing the Lagrangian angle $\phi$, the above gives

$$
\mu_{2}=\frac{i \bar{\partial} \phi}{1-i \bar{\mu}_{1} \bar{\partial} \phi} .
$$

By derivation of the above with respect of $\mu_{1}$ we obtain

$$
i \partial \bar{\partial} \phi=\rho_{0}, \quad-i \partial \bar{\partial} \phi=\bar{\rho}_{0} .
$$

The Lagrangian condition $\rho_{0}=\bar{\rho}_{0}$ implies that $\rho_{0}=0$ and therefore $\mu_{2}$ is anti-holomorphic, which means that $\Sigma$ is locally orthogonal to a flat surface in $\mathrm{H}^{3}$.

Because of $\rho_{0}=0$ we obtain

$$
\partial \bar{\partial} \phi=0
$$

The fact that $\mu_{2}$ is an anti-holomorphic function of $\mu_{1}$ implies that $\ln \bar{\sigma}_{0}$ is anti-holomorphic too, which means that $\partial \ln \bar{\sigma}_{0}=0$ and then

$$
\partial \ln \left|\sigma_{0}\right|=2 i \partial \phi
$$

The expression of $\sigma_{0}$ in terms of $\phi$ is

$$
\sigma_{0}=\frac{\partial \bar{\mu}_{2}}{\left(1+\mu_{1} \bar{\mu}_{2}\right)^{2}}=-(\partial \phi)^{2}-i \partial^{2} \phi .
$$

Then we have

$$
\left|\sigma_{0}\right| e^{2 i \phi}=-(\partial \phi)^{2}-i \partial^{2} \phi
$$

which gives

$$
\left|\sigma_{0}\right| e^{i \phi}=\left[-(\partial \phi)^{2}-i \partial^{2} \phi\right] e^{-i \phi}
$$

and therefore $\left|\sigma_{0}\right|=e^{-i \phi} \partial^{2} e^{-i \phi}$, which implies equation (4.6). 
We now prove the converse. If $\phi$ is a real solution of (4.6), it satisfies

$$
-i \partial^{2} \phi-(\partial \phi)^{2}=\left|\sigma_{0}\right| e^{2 i \phi}=\sigma_{0} .
$$

By the assumption that $\Sigma$ is locally orthogonal to a flat in $\mathrm{H}^{3}, \mu_{2}$ is antiholomorphic and therefore $\sigma_{0}$ is a holomorphic function. Then

$$
\bar{\partial}\left[-i \partial^{2} \phi-(\partial \phi)^{2}\right]=0,
$$

which implies

$$
\partial\left[(\bar{\partial} \partial \phi) e^{-2 i \phi}\right]=0,
$$

and hence there is a holomorphic function $\beta$ such that

$$
(\bar{\partial} \partial \phi) e^{-2 i \phi}=\beta, \quad(\bar{\partial} \partial \phi) e^{2 i \phi}=\bar{\beta} .
$$

Therefore, the function $\phi$ can be written as

$$
\phi=a+\bar{a},
$$

where $a$ is a holomorphic function. In other words, we have proved that $\bar{\partial} \partial \phi=$ 0 .

On the other hand, using equations (4.11) and (4.12), $\mu_{2}$ must satisfies the following equation:

$$
-i \partial^{2} a-(\partial a)^{2}=\frac{\partial \bar{\mu}_{2}}{\left(1+\mu_{1} \bar{\mu}_{2}\right)^{2}},
$$

but because of the fact that $\bar{\mu}_{2}$ and $a$ are holomorphic, the above equation is equivalent to an ordinary differential equation of first order. In addition, we observe that

$$
\bar{\mu}_{2}=-\frac{i \partial a}{1+i \mu_{1} \partial a}
$$

is a solution of (4.13) and because this equation is equivalent to an ODE of first order, it is unique.

Then it is easy to see that $H^{\mu_{1}}=0$ and therefore $\Sigma$ is a Lagrangian area stationary surface.

In the following proposition we give an explicit local expression of all Lagrangian area stationary surfaces in $\mathrm{L}\left(\mathrm{H}^{3}\right)$ in terms of the holomorphic coordinates $\left(\mu_{1}, \mu_{2}\right)$ on $\mathrm{P}^{1} \times \mathrm{P}^{1}-\bar{\Delta}$ :

PROPOSITION 9. Every Lagrangian area stationary surface $\Sigma$ in $\mathrm{L}\left(\mathrm{H}^{3}\right)$ of rank two can be locally parameterized by

$$
\Sigma \rightarrow \mathrm{L}\left(\mathrm{H}^{3}\right):\left(\mu_{1}, \bar{\mu}_{1}\right) \mapsto\left(\mu_{1}, \mu_{2}=\frac{\bar{\lambda}_{1} \bar{\mu}_{1}+1}{\bar{\mu}_{1}+\bar{\lambda}_{2}}\right),
$$

where $\lambda_{1}, \lambda_{2} \in \mathrm{C}$ with $\lambda_{1} \lambda_{2} \neq 1$. 
Proof. Let $\Sigma$ be a Lagrangian area stationary surface of rank two in $\mathrm{L}\left(\mathrm{H}^{3}\right)$. By Proposition 8 the surface $\Sigma$ is locally orthogonal to a flat surface in $\mathrm{H}^{3}$, which allows us to obtain the holomorphic parameterization $\left(\mu_{1}, \bar{\mu}_{1}\right) \mapsto$ $\left(\mu_{1}, \mu_{2}\left(\bar{\mu}_{1}\right)\right)$. In addition, the Lagrangian angle $\phi$ must satisfies equation (4.6).

There is a holomorphic function $a$ such that $\phi=a+\bar{a}$, and applying this to equation (4.6), we get:

$$
e^{3 i \bar{a}} \bar{\partial}^{2} e^{i \bar{a}}=e^{-3 i a} \partial^{2} e^{-i a}=c_{0},
$$

where $c_{0} \in \mathrm{R}$ is a real constant.

Then the holomorphic function $a$ satisfies

$$
\partial^{2} e^{-i a}=c_{0} e^{3 i a},
$$

which is equivalent to the following ordinary differential equation of second order:

$$
\ddot{x}=c_{0} x^{-3} .
$$

The unique solution of (4.15) is

$$
a=\frac{i}{2} \log \left[\left(\alpha_{0} \mu_{1}+\beta_{0}\right)^{2}-c_{0}\right]-\frac{i}{2} \log \alpha_{0},
$$

and the Lagrangian angle is $\phi=a+\bar{a}$.

The immersion of $\Sigma$ is obtained by substituting (4.16) into (4.7) and then

$$
\mu_{2}=\frac{i \bar{\partial} \phi}{1-i \bar{\mu}_{1} \bar{\partial} \phi}=\frac{i \bar{\partial} \bar{a}}{1-i \bar{\mu}_{1} \bar{\partial} \bar{a}}=\frac{\alpha_{0}^{2} \bar{\mu}_{1}+\alpha_{0} \beta_{0}}{\alpha_{0} \beta_{0} \bar{\mu}_{1}+\beta_{0}^{2}-c_{0}} .
$$

If we set $\bar{\lambda}_{1}=\alpha_{0} \beta_{0}^{-1}$ and $\bar{\lambda}_{2}=\left(\beta_{0}^{2}-c_{0}\right)\left(\alpha_{0} \beta_{0}\right)^{-1}$ then the area stationary surface $\Sigma$ is given by the immersion (4.14).

If $\lambda_{1} \lambda_{2}=1$ we find that $\Sigma$ is a totally null surface given by the immersion $\mu_{2}=\lambda_{1}$, and so it is not of rank two.

For a given Lagrangian area stationary surface $\Sigma$ in $\mathrm{L}\left(\mathrm{H}^{3}\right)$, there is locally a family of parallel flat surfaces in $\mathrm{H}^{3}$ such that their oriented normals are contained in $\Sigma$. We recall the classification of complete flat surfaces in hyperbolic 3-space:

PROPOSITION 10 ([14], [15]). Let $S$ be a complete flat surface in hyperbolic 3 -space $\mathrm{H}^{3}$. Then $S$ is either a horosphere or an equidistant tube of a geodesic in $\mathrm{H}^{3}$.

To proof the main theorem we need to introduce a particular class of surface in hyperbolic 3-space $\mathrm{H}^{3}$ : 
Definition 4. A surface $S$ in hyperbolic 3-space $\mathrm{H}^{3}$ is called isoparametric if the principal curvatures of $S$ are constant.

Note that all parallel surfaces $\left\{S_{t}\right\}_{t \in I}$ to the isoparametric surface $S$ are also isoparametric.

The following proposition gives a classification of the isoparametric surfaces in hyperbolic 3-space:

Proposition 11 ([3]). Let $S$ be an isoparametric surface in $\mathrm{H}^{3}$. Then $S$ is either a totally geodesic hyperbolic 2-space, or a totally umbilical surface or an equidistant tube around a geodesic.

We now prove our main result:

THEOREM 3. Let $S \subset \mathrm{H}^{3}$ be a $C^{3}$ smooth immersed oriented surface and $\Sigma \subset \mathrm{L}\left(\mathrm{H}^{3}\right)$ be the Lagrangian surface formed by the oriented geodesics normal to $S$.

The surface $\Sigma$ is area stationary iff $S$ is an equidistant tube around a geodesic.

PROOF. Let $\Sigma$ be a Lagrangian geodesic congruence formed by the oriented geodesics normal to $S$.

First assume that $\Sigma$ is area stationary. Since it cannot be of rank 0 , as that would mean that it is totally null, and by Proposition 5 it cannot be of rank 1 , we conclude that $\Sigma$ is of rank two. Thus it is given locally by the graph:

$$
\bar{\mu}_{2}=\frac{1+\lambda_{1} \mu_{1}}{\mu_{1}+\lambda_{2}},
$$

where $\lambda_{1}, \lambda_{2} \in \mathrm{C}$. 1 .

The non-degeneracy condition of the induced metric $\mathrm{G}_{\Sigma}$ implies that $\lambda_{1} \lambda_{2} \neq$

In this case, an orthogonal surface $S \subset \mathrm{H}^{3}$ can be obtained by solving the following differential equation

$$
2 \partial r=\frac{\partial \mu_{2}}{\mu_{2}\left(1+\bar{\mu}_{1} \mu_{2}\right)}+\frac{\partial \bar{\mu}_{2}}{\bar{\mu}_{2}\left(1+\mu_{1} \bar{\mu}_{2}\right)}+\frac{\bar{\mu}_{2}}{1+\mu_{1} \bar{\mu}_{2}},
$$

and, by using the fact that $\mu_{2}$ is holomorphic, we obtain, after a brief computation, that

$$
2 \partial r=\frac{\lambda_{1}}{1+\lambda_{1} \mu_{1}},
$$

which implies

$$
r=\frac{1}{2} \log \left|1+\lambda_{1} \mu_{1}\right|^{2}+r_{0}
$$


The function $\Delta$, given by (4.3), is:

$$
\Delta=\frac{4\left|\lambda_{1}\right|^{2}\left[e^{-2 r_{0}}-\left|\lambda_{1} \lambda_{2}-1\right|^{2} e^{2 r_{0}}\right]}{\left|\left(\lambda_{1} \mu_{1}+1\right)^{2}+\lambda_{1} \lambda_{2}-1\right|^{2}} .
$$

The optical scalars $\rho$ and $\sigma$ of the Lagrangian area stationary surface $\Sigma$ given by (4.1) and (4.2) are:

$$
\sigma=\frac{2\left(\lambda_{1} \lambda_{2}-1\right)}{e^{-2 r_{0}}-\left|\lambda_{1} \lambda_{2}-1\right|^{2} e^{2 r_{0}}} \cdot \frac{1+\bar{\lambda}_{1} \bar{\mu}_{1}}{1+\lambda_{1} \mu_{1}}
$$

and

$$
\rho=-1+\frac{2}{1-e^{4 r_{0}}\left|\lambda_{1} \lambda_{2}-1\right|^{2}} .
$$

If we denote by $h$ the mean curvature of the surface $S \subset \mathrm{H}^{3}$, Proposition 2 gives:

$$
h=1+\frac{2}{e^{4 r_{0}}\left|\lambda_{1} \lambda_{2}-1\right|^{2}-1} .
$$

Consider now the principal curvatures $m_{1}$ and $m_{2}$ of the surface $S$. The fact that $S$ is flat means that $m_{1} m_{2}=1$. Then the mean curvature of the surface $S$ is

$$
h=\frac{m_{1}+m_{2}}{2}=\frac{m_{1}+m_{1}^{-1}}{2},
$$

and by using the relation (4.18), we observe that $m_{1}$ must satisfy the following quadratic equation

$$
m_{1}^{2}-2\left(1+\frac{2}{e^{4 r_{0}}\left|\lambda_{1} \lambda_{2}-1\right|^{2}-1}\right) m_{1}+1=0 .
$$

Therefore the principal curvatures of the surface $S$ are constant and in particular are given by:

$$
m_{1}=\frac{e^{2 r_{0}}\left|\lambda_{1} \lambda_{2}-1\right|+1}{e^{2 r_{0}}\left|\lambda_{1} \lambda_{2}-1\right|-1}, \quad m_{2}=\frac{e^{2 r_{0}}\left|\lambda_{1} \lambda_{2}-1\right|-1}{e^{2 r_{0}}\left|\lambda_{1} \lambda_{2}-1\right|+1},
$$

and hence the surface $S$ is isoparametric. Propositions 10 and 11 tell us that the surface $S$ can be either a horosphere or an equidistant tube around a geodesic. By previous work (see the papers [5] and [6]) we have seen that geodesic congruences orthogonal to horospheres are totally null (the induced metric is degenerate). Therefore the surface $S$ must be an equidistant tube around a geodesic $\gamma$. 
In fact, every area stationary surface $\Sigma$ is orthogonal to the set $\left\{S_{r_{0}}\right\}_{r_{0} \in \mathrm{R}}$ of all parallel equidistant tubes around a geodesic $\gamma$ and each such a surface $S_{r_{0}}$ is of hyperbolic distance $r_{0}$ from the surface $S_{0}$.

Conversely, assume that the surface $S \subset \mathrm{H}^{3}$ is an equidistant tube around a geodesic $\gamma^{\prime}$ with holomorphic coordinates $\left(\mu_{1}=\mu_{1}^{\prime}, \mu_{2}=\mu_{2}^{\prime}\right)$. Then $S$ belongs to the set of all parallel equidistant tubes $\mathscr{U}_{\gamma^{\prime}}=\left\{S_{r_{0}}\right\}_{r_{0} \in \mathrm{R}}$ around the geodesic $\gamma^{\prime}$. We first find an explicit expression of the orthogonal geodesic congruence $\Sigma \subset \mathrm{L}\left(\mathrm{H}^{3}\right)$ to all surfaces in $\mathcal{U}_{\gamma^{\prime}}$.

Consider the hyperbolic 3 -space $\mathrm{H}^{3}$ in the half space model with local coordinates $\left(x_{0}, x_{1}, x_{2}\right)$.

By direct computation we get that for a given point $p=\left(p_{0}, p_{1}, p_{2}\right)$ in $\mathrm{H}^{3}$ and a given vector $e_{0}=a_{0} \frac{\partial}{\partial x_{0}}+a_{1} \frac{\partial}{\partial x_{1}}+a_{2} \frac{\partial}{\partial x_{2}} \in T_{p} \mathrm{H}^{3}$ the unique geodesic $\gamma: I \subset \mathrm{R} \rightarrow \mathrm{H}^{3}: r \mapsto \gamma(r) \in \mathrm{H}^{3}$ such that

$$
\gamma(0)=p, \quad \dot{\gamma}(0)=e_{0},
$$

where $I$ is an open interval containing 0 and the dot denotes the differentiation with respect of $r$, is defined by:

$$
\begin{aligned}
& x_{0}=p_{0} \sqrt{\frac{a_{0}^{2}+a_{1}^{2}+a_{2}^{2}}{a_{1}^{2}+a_{2}^{2}}} \operatorname{sech}\left[\frac{\sqrt{a_{0}^{2}+a_{1}^{2}+a_{2}^{2}}}{p_{0}}\left(r+r_{0}\right)\right], \\
& x_{1}=\frac{a_{1} p_{0} \sqrt{a_{0}^{2}+a_{1}^{2}+a_{2}^{2}}}{a_{1}^{2}+a_{2}^{2}} \tanh \left[\frac{\sqrt{a_{0}^{2}+a_{1}^{2}+a_{2}^{2}}}{p_{0}}\left(r+r_{0}\right)\right]+c_{3} \text {, } \\
& x_{2}=\frac{a_{2} p_{0} \sqrt{a_{0}^{2}+a_{1}^{2}+a_{2}^{2}}}{a_{1}^{2}+a_{2}^{2}} \tanh \left[\frac{\sqrt{a_{0}^{2}+a_{1}^{2}+a_{2}^{2}}}{p_{0}}\left(r+r_{0}\right)\right]+c_{4} .
\end{aligned}
$$

Introduce complex coordinate $z=x_{1}+i x_{2}$ and set $t=x_{0}$. We then obtain

$$
\xi=c_{1}+i c_{2}=\frac{\beta}{t_{0}^{2}}, \quad \eta=c_{3}+i c_{4}=z_{0}+t_{0} \frac{a}{\bar{\beta}},
$$

where $t_{0}=t(0), z_{0}=z(0), \beta=a_{1}+i a_{2}$ and $a=a_{0}$.

Therefore for a given point $p=\left(z_{0}, t_{0}\right)$ and a given vector $e_{0}=a \frac{\partial}{\partial t}+$ $\beta \frac{\partial}{\partial z}+\bar{\beta} \frac{\partial}{\partial \bar{z}}$ the unique oriented geodesic $\gamma=(\xi, \eta)$ with the initial conditions $\gamma(0)=p$ and $\dot{\gamma}(0)=e_{0}$ is given by

$$
\xi=\frac{\beta}{t_{0}^{2}}, \quad \eta=z_{0}+t_{0} \frac{a}{\bar{\beta}}
$$


Fix the point $p$ on the given oriented geodesic $\gamma^{\prime}=\left(\xi^{\prime}, \eta^{\prime}\right)$. Let $\gamma=(\xi, \eta)$ be an oriented geodesic that intersects $\gamma^{\prime}$ orthogonally at $p$. Denote the unit tangent vectors of $\gamma, \gamma^{\prime}$ at $p$ by $e_{0}, e_{0}^{\prime}$ respectively. The orthogonality condition gives the following relation:

$$
e_{0}=\frac{1}{\sqrt{2}}\left(e^{-i \theta} e_{+}^{\prime}+e^{i \theta} e_{-}^{\prime}\right),
$$

for some $\theta \in[0,2 \pi)$ where

$$
e_{+}^{\prime}=\frac{1}{\sqrt{2}\left|\xi^{\prime}\right| \cosh ^{2} r_{0}} \frac{\partial}{\partial t}+\frac{1}{\sqrt{2} \cosh ^{2} r_{0}}\left(-\frac{e^{-r_{0}}}{\bar{\xi}^{\prime}} \frac{\partial}{\partial z}+\frac{e^{r_{0}}}{\xi^{\prime}} \frac{\partial}{\partial \bar{z}}\right), \quad e_{-}^{\prime}=\bar{e}_{+}^{\prime} .
$$

Thus the unit tangent vector of $\gamma$ is

$$
e_{0}=\frac{\cos \theta}{\left|\xi^{\prime}\right| \cosh ^{2} r_{0}} \frac{\partial}{\partial t}+\frac{\sinh \left(r_{0}+i \theta\right)}{\bar{\xi}^{\prime} \cosh ^{2} r_{0}} \frac{\partial}{\partial z}+\frac{\sinh \left(r_{0}-i \theta\right)}{\xi^{\prime} \cosh ^{2} r_{0}} \frac{\partial}{\partial \bar{z}} .
$$

Applying (4.20), the oriented geodesic $\gamma=(\xi, \eta)$ is

$$
\xi=\xi^{\prime} \sinh \left(r_{0}+i \theta\right), \quad \eta=\eta^{\prime}+\frac{1}{\bar{\xi}^{\prime} \tanh \left(r_{0}-i \theta\right)}
$$

Moving the point $p$ along the geodesic $\left(\xi^{\prime}, \eta^{\prime}\right)$, it is equivalent to an affine shift of $r_{0}$.

Therefore we obtain the surface $\Sigma$ given by the immersion $f: \mathrm{C} \rightarrow \mathrm{L}\left(\mathrm{H}^{3}\right)$ : $(\nu, \bar{v}) \mapsto(\xi(\nu, \bar{v}), \eta(\nu, \bar{v}))$ where

$$
\xi=\xi^{\prime} \sinh v, \quad \eta=\eta^{\prime}+\frac{1}{\bar{\xi}^{\prime} \tanh \bar{v}},
$$

with $v=r+i \theta$.

If we change the coordinates from $(\xi, \eta)$ to holomorphic coordinates $\left(\mu_{1}\right.$, $\mu_{2}$ ) on $\mathrm{L}\left(\mathrm{H}^{3}\right)$, the surface $\Sigma$ is given by the following immersion

$$
\begin{aligned}
& \mu_{1}(v, \bar{v})=\frac{1-\cosh \bar{v}-\eta^{\prime} \bar{\xi}^{\prime} \sinh \bar{v}}{\bar{\xi}^{\prime} \sinh \bar{v}}, \\
& \mu_{2}(v, \bar{v})=\frac{\xi^{\prime} \sinh v}{1+\cosh v+\bar{\eta}^{\prime} \xi^{\prime} \sinh v} .
\end{aligned}
$$

We can easily see that

$$
\sinh v=\frac{2\left(\xi^{\prime}\right)^{-1} \mu_{2}}{1+\bar{\mu}_{1} \mu_{2}} \quad \text { and } \quad \cosh v=\frac{1-\bar{\mu}_{1} \mu_{2}-2 \bar{\eta}^{\prime} \mu_{2}}{1+\bar{\mu}_{1} \mu_{2}},
$$


and from the identity $\cosh ^{2} v-\sinh ^{2} v=1$ we find that the Lagrangian surface $\Sigma$ is an area stationary surface since it can be written

$$
\bar{\mu}_{2}=\frac{\lambda_{1} \mu_{1}+1}{\mu_{1}+\lambda_{2}}, \quad \text { with } \quad \lambda_{1}=\frac{1}{\eta^{\prime}}, \quad \lambda_{2}=\frac{1}{\eta^{\prime}}\left[\left(\eta^{\prime}\right)^{2}-\frac{1}{\left(\bar{\xi}^{\prime}\right)^{2}}\right],
$$

which completes the proof.

Note: We have proved on the main theorem that every area stationary Lagrangian surface is given by the graph (4.14) and is orthogonal to a family of parallel equidistant tubes $\left\{S_{t}\right\}_{t \in I}$ around to the following oriented geodesics $\gamma^{\prime}=\left(\mu_{1}^{\prime}, \mu_{2}^{\prime}\right)$ and $\tilde{\gamma}^{\prime}=\left(\tilde{\mu}_{1}^{\prime}, \tilde{\mu}_{2}^{\prime}\right)$, given by

$$
\mu_{1}^{\prime}=\frac{-1+\sqrt{1-\lambda_{1} \lambda_{2}}}{\lambda_{1}}, \quad \mu_{2}^{\prime}=\frac{\bar{\lambda}_{1}}{1+\sqrt{1-\bar{\lambda}_{1} \bar{\lambda}_{2}}},
$$

and

$$
\tilde{\mu}_{1}^{\prime}=-\frac{1+\sqrt{1-\lambda_{1} \lambda_{2}}}{\lambda_{1}} \quad \tilde{\mu}_{2}^{\prime}=\frac{\bar{\lambda}_{1}}{1-\sqrt{1-\bar{\lambda}_{1} \bar{\lambda}_{2}}} .
$$

Consider now the antipodal map $\tau: \mathrm{P}^{1} \rightarrow \mathrm{P}^{1}: x \mapsto-\bar{x}^{-1}$ and observe that $\tilde{\mu}_{1}^{\prime}=\tau\left(\mu_{2}^{\prime}\right)$ and $\tilde{\mu}_{2}^{\prime}=\tau\left(\mu_{1}^{\prime}\right)$ which means that the geodesic $\tilde{\gamma}$ is obtained by reversing the orientation of the geodesic $\gamma$. In other words $\tilde{\gamma}$ and $\gamma$ describe the same geodesic, up to orientation.

\section{Rotationally symmetric maximal graphs}

We now investigate area stationary surfaces of rank two that are not Lagrangian or holomorphic. The simplest case is the rotationally symmetric ones, see also [9].

DEFINITION 5. A surface in $\mathrm{L}\left(\mathrm{H}^{3}\right)$ is rotationally symmetric if it is invariant under the induced isometry of $\mathrm{G}$.

Lemma 1. A graph $\Sigma \rightarrow \mathrm{L}\left(\mathrm{H}^{3}\right): \mu_{1} \mapsto\left(\mu_{1}, \mu_{2}=F\left(\mu_{1}, \bar{\mu}_{1}\right)\right)$ is rotationally symmetric if and only if $F\left(\mu_{1}, \bar{\mu}_{1}\right)=G(R) e^{i \theta}$ for some complex-valued function $G$, where $\mu_{1}=R e^{i \theta}$.

To find a complete characterisation of all area stationary rotationally symmetric surfaces in $\mathrm{L}\left(\mathrm{H}^{3}\right)$ of rank two, seems to be a very difficult problem because the correspondent ordinary differential equations are too complicated. We then investigate the case of $\operatorname{Re} G=0$ :

Proposition 12. Consider a rank two surface $\Sigma$ immersed by $\mu_{1}=R e^{i \theta}$ and $F\left(\mu_{1}, \bar{\mu}_{1}\right)=i A e^{i \theta}$, where $A=A(R)$ is real-valued function. Then 
the surface $\Sigma$ is a holomorphic curve iff $A=c_{0} R$, while is Lagrangian iff $A=c_{0} R^{-1}$.

The following proposition gives a two parameter family of rotationally symmetric surfaces of rank two in $\mathrm{L}\left(\mathrm{H}^{3}\right)$ that are area stationary:

Proposition 13. Consider the rotationally symmetric $\Sigma \subset \mathrm{L}\left(\mathrm{H}^{3}\right)$ of rank two, given locally immersed by $\mu_{1}=R e^{i \theta}$ and $\mu_{2}=F\left(\mu_{1}, \bar{\mu}_{1}\right)=i Z^{1 / 2} e^{i \theta}$, where $Z=Z(R)$ is a real-valued function.

Then $\Sigma$ is area stationary if and only if the function $Z$ is of the form:

$$
Z(R)=c+\frac{b R^{2}}{c R^{2}-1},
$$

where $b$ and $c$ are real constants that aren't both 0 .

Proof. We first compute the functions $\Delta, \lambda$ and $\sigma$ of the rotationally symmetric surface $\Sigma$. Since the surface $\Sigma$ is of rank two, then by Proposition 6, it is area stationary iff it satisfies the equation (4.4) which implies that the function $Z$ is satisfying the following ordinary differential equation:

$$
R\left(R^{2} Z-1\right) \ddot{Z}-2 R^{3} \dot{Z}^{2}+\left(3 R^{2} Z+1\right) \dot{Z}=0,
$$

where the dot denotes differentiation with respect of the real variable $R$.

We first observe that the expression of the real-valued function $Z=Z(R)$ given in (5.1) satisfies the ordinary differential equation (5.2). Furthermore, we have obtained a two parameter family of such solutions depended of the constants $b$ and $c$, which implies that the functions $Z=Z(R)$ given in (5.1) form the complete solutions of (5.2).

Note that for every choice of $b, c \in \mathrm{R}$, the area stationary rotationally symmetric surface $\Sigma_{b c}$, immersed by $\mu_{1}=R e^{i \theta}$ and $\mu_{2}=F\left(\mu_{1}, \bar{\mu}_{1}\right)=i Z^{1 / 2} e^{i \theta}$, where $Z=Z(R)$ is given by (5.1), is neither Lagrangian nor holomorphic curve, since for

$$
Z=A^{2}=c_{0}^{2} R^{2} \quad \text { or } \quad Z=A^{2}=c_{0}^{2} R^{-2},
$$

are not solutions of the ordinary differential equation (5.2).

We now investigate the induced metric of area stationary rotationally symmetric surfaces. Such a surface $\Sigma$ can be given locally by the immersion $\mu_{1}=R e^{i \theta}, \mu_{2}=i z^{1 / 2} e^{i \theta}$, where $z$ is given by (5.1).

PROPOSITION 14. Let $\Sigma$ be an area stationary rotationally symmetric surface in $\mathrm{L}\left(\mathrm{H}^{3}\right)$. Such a surface can be determined by the constants $b, c \in \mathrm{R}(b \neq 0)$, given by (5.1). 
If $b>0$ the induced metric $\mathrm{G}_{\Sigma}$ is Lorentz, while for $b<0$ the surface $\Sigma$ has at least two degenerate curves.

Proof. From Theorem 1 we need to find the expression $|\sigma|^{2}-\lambda^{2}$. After a brief computation we find that

$$
|\sigma|^{2}-\lambda^{2}=A\left[b R^{4}+\left(c R^{2}-1\right)^{2}\right]\left[\left(\left(c^{2}+b\right) R^{2}-1\right)^{2}+b\right],
$$

where $A=64 \Delta^{-2}\left|1+\mu_{1} \bar{\mu}_{2}\right|^{-2}\left(1+R^{2} z\right)^{-2}\left(c R^{2}-1\right)^{-4}>0$.

Observe that $|\sigma|^{2}-\lambda^{2}>0$ for $b>0$ and therefore the rotationally symmetric area stationary surface $\Sigma$ is Lorentzian.

We consider the case of $b=-k^{2}<0$ and $c^{2}+b>0$. Then the induced metric $\mathrm{G}_{\Sigma}$ of the surface $\Sigma$ is degenrate at the following least four curves,

$$
\begin{array}{ll}
R= \pm(c-k)^{-1 / 2}, & R= \pm \sqrt{\frac{k+1}{c^{2}-k^{2}}}, \quad \text { for } k>-1, \\
R= \pm(c-k)^{-1 / 2}, & R= \pm \sqrt{\frac{1-k}{c^{2}-k^{2}}}, \quad \text { for } k<1, \\
R= \pm(c-k)^{-1 / 2}, & R= \pm \sqrt{\frac{k+1}{c^{2}-k^{2}}}, \quad R= \pm \sqrt{\frac{1-k}{c^{2}-k^{2}}}, \quad \text { for }|k|<1 .
\end{array}
$$

In case of $b=-k^{2}<0$ and $c^{2}+b<0$ the induced metric $\mathrm{G}_{\Sigma}$ is degenerate at the following least two curves,

$$
\begin{array}{ll}
R= \pm(c+k)^{-1 / 2}, & R= \pm \sqrt{\frac{k+1}{c^{2}-k^{2}}}, \quad \text { for } k<-1, \\
R= \pm(c+k)^{-1 / 2}, & R= \pm \sqrt{\frac{1-k}{c^{2}-k^{2}}}, \quad \text { for } k>1, \\
R= \pm(c+k)^{-1 / 2}, & R= \pm \sqrt{\frac{k+1}{c^{2}-k^{2}}}, \quad R= \pm \sqrt{\frac{1-k}{c^{2}-k^{2}}}, \quad \text { for }|k|>1 . \\
R= \pm(c+k)^{-1 / 2}, & \text { for }|k|<1,
\end{array}
$$

and the proposition follows.

We know that $\mathrm{L}\left(\mathrm{H}^{3}\right)$ can be identified with $\mathrm{P}^{1} \times \mathrm{P}^{1}-\bar{D}$, where $\bar{D}=$ $\left\{(x, y) \in \mathrm{P}^{1} \times \mathrm{P}^{1}: y=-\bar{x}^{-1}\right\}$ is the reflected diagonal of $\mathrm{P}^{1}$. We would like to see whether an area stationary rotationally symmetric surface $\Sigma$ intersects $\bar{D}$. 
To do this we consider the local immersion $\mu_{1}=R e^{i \theta}$ and $\mu_{2}=i Z^{1 / 2} e^{i \theta}$, of $\Sigma$, where $Z=Z(R)$ is given by (5.1). Assume that a point $(R, \theta) \in \Sigma$ is very near to $\bar{D}$. Then the equation $\mu_{1}=-\bar{\mu}_{2}^{-1}$ gives the following quadratic equation

$$
\left(c^{2}+b\right) R^{4}-1=0 .
$$

Then, we conclude that for $b$ positive the Lorentzian area stationary rotationally symmetric surface $\Sigma$ must be an open subset of $\mathrm{L}\left(\mathrm{H}^{3}\right)$.

Consider the surface $T \subset \mathrm{L}\left(\mathrm{H}^{3}\right)$ locally parameterised by

$$
\mu_{1}(R, \theta)=R e^{i \theta}, \quad \mu_{2}(R, \theta)= \pm i\left(c+\frac{b R^{2}}{c R^{2}-1}\right)^{1 / 2} e^{i \theta} .
$$

In order $T$ to be an immersed complete surface we require $c<0$. If in addition we assume that $T$ must be a closed surface, we require $c^{2}+b<0$. We can see now that for $R=0$ and $R \rightarrow \infty$, we obtain the following two curves on $T$ :

$$
\mu_{2}=i \sqrt{c} e^{i \theta} \quad \text { and } \quad \mu_{2}=i \sqrt{\frac{c^{2}+b}{c}} e^{i \theta} .
$$

Under the $i$-th projection $\mathrm{L}\left(\mathrm{H}^{3}\right)=\mathrm{S}^{2} \times \mathrm{S}^{2}-D \rightarrow \mathrm{S}^{2}$ for $i=1,2$ we observe that these surfaces $T$ double cover the sphere, except the north and south pole, where the inverse image of each of these points, is a circle. Therefore, the surface $T$ must be an area stationary tori in $\mathrm{L}\left(\mathrm{H}^{3}\right)$.

AcKNOWLEDGEMEnT. The author would like to thank Brendan Guilfoyle, Henri Anciaux and Stelios Georgiou for many stimulating and helpful conversations.

\section{REFERENCES}

1. Alekseevsky, D. V., Guilfoyle, B., and Klingenberg, W., On the geometry of spaces of oriented geodesics, Ann. Global Anal. Geom. 40 (2011), 389-409.

2. Anciaux, H., Guilfoyle, B., and Romon, P., Minimal submanifolds in the tangent bundle of a Riemannian surface, J. Geom. Phys. 61 (2011), 237-247.

3. Cartan, E., Familles de surfaces isoparamétriques dans les espaces à courbure constante, Ann. Mat. Pura Appl. 17 (1938), 177-191.

4. Georgiou, N., and Guilfoyle, B., On the space of oriented geodesics of hyperbolic 3-space, Rocky Mountain J. Math. 40 (2010), 1183-1219.

5. Georgiou, N., Guilfoyle, B., and Klingenberg, W., Totally null surfaces in neutral Kähler 4-manifolds, preprint 2008 [math.DG/0810.4054].

6. Georgiou, N., and Guilfoyle, B., A characterization of Weingarten surfaces in hyperbolic 3-space, Abh. Math. Sem. Hambg. 80 (2010), 233-253.

7. Guilfoyle, B., and Klingenberg, W., An indefinite Kähler metric on the space of oriented lines, J. London Math. Soc. (2) 72 (2005), 497-509. 
8. Guilfoyle, B., and Klingenberg, W., A neutral Kähler metric on the space of time-like lines in Lorentzian 3-space, preprint 2005 [math.DG/0608782].

9. Guilfoyle, B., and Klingenberg, W., Area-stationary surfaces in neutral Kähler 4-manifolds, Beiträge Algebra Geom. 49 (2008), 481-490.

10. Guilfoyle, B., and Klingenberg, W., Proof of the Carathéodory conjecture by mean curvature flow in the space of oriented affine lines, preprint 2008 [math.DG/0808.0851].

11. Hitchin, N. J., Monopoles and geodesics, Comm. Math. Phys. 83 (1982), 579-602.

12. Salvai, M., On the geometry of the space of oriented lines in Euclidean space, Manuscripta Math. 118 (2005), 181-189.

13. Salvai, M., On the geometry of the space of oriented lines of the hyperbolic space, Glasg. Math. J. 49 (2007), 357-366.

14. Sasaki, S., On complete flat surfaces in hyperbolic 3-space, Ködai Math. Sem. Rep. 25 (1973), 449-457.

15. Volkov, Ju. A., and Vladimirova, S. M., Isometric immersions of the Euclidean plane in Lobačevskii space (Russian), Mat. Zametki 10(1971), 327-332, English translation: Math. Notes 10 (1971), 655-661.

16. Weierstrass, K., Untersuchungen über die Flächen, deren mittlere Krümmung überall gleich Null ist, Monatsber. Akad. Wiss. Berlin 1866, 612-625, cf. Math. Werke III, 39-52.

17. Whittaker, E. T., On the partial differential equations of mathematical physics, Math. Ann. 57 (1903), 333-355.

UNIVERSIDADE DE SÃO PAULO

IME, BLOCO A

1010 RUA DO MATÃO

CIDADE UNIVERSITÁRIA

05508-090 SÃO PAULO

BRAZIL

E-mail: nikos@ime.usp.br 